\title{
Explicitly representing the semantics of composite positional tolerance for patterns of holes
}

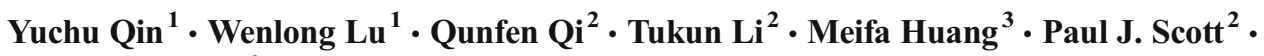 \\ Xiangqian Jiang ${ }^{2}$
}

Received: 10 May 2016/Accepted: 5 September 2016/Published online: 10 October 2016

(C) The Author(s) 2016. This article is published with open access at Springerlink.com

\begin{abstract}
Representing the semantics of the interaction of two or more tolerances (i.e., composite tolerance) explicitly to make them computer-understandable is currently a challenging task in computer-aided tolerancing (CAT). We have proposed a description logic (DL) ontology-based approach to complete this task recently. In this paper, the representation of the semantics of the composite positional tolerance (CPT) for patterns of holes (POHs) is used as an example to illustrate the proposed approach. This representation mainly includes representing the structure knowledge of the CPT for POHs in DL terminological axioms; expressing the constraint knowledge with Horn rules; and describing the individual knowledge using DL assertional axioms. By implementing the representation with the web ontology language (OWL) and the semantic web rule language (SWRL), a CPT ontology is developed. This ontology has explicitly computerunderstandable semantics due to the logic-based semantics of OWL and SWRL. As is illustrated by an engineering example, such semantics makes it possible to automatically check the consistency, reason out the new knowledge, and
\end{abstract}

Qunfen Qi

q.qi@hud.ac.uk

1 The State Key Laboratory of Digital Manufacturing Equipment and Technology, School of Mechanical Science and Engineering, Huazhong University of Science and Technology, Wuhan 430074, People's Republic of China

2 EPSRC Centre for Innovative Manufacturing in Advanced Metrology, School of Computing and Engineering, University of Huddersfield, Huddersfield HD1 3DH, UK

3 School of Mechanical and Electrical Engineering, Guilin University of Electronic Technology, Guilin 541004, People's Republic of China implement the semantic interoperability of CPT information. Benefiting from this, the ontology provides a semantic enrichment model for the CPT information extracted from CAD/ CAM systems.

Keywords Tolerance semantics · Semantic representation · Composite positional tolerance $\cdot$ Pattern of holes $\cdot$ Tolerance modeling · Ontology

\section{Introduction}

The emerge of geometric dimensioning and tolerancing (GD\&T) brings the idea that part features should be controlled in the geometric characteristics of size, form, orientation, location, run-out, and surface texture [1]. This entails various GD\&T-related research topics like tolerance modeling, tolerance specification, tolerance analysis, tolerance allocation, tolerance transfer, and tolerance evaluation, where tolerance modeling is seen as one of the hottest topics. Tolerance modeling mainly concerns how to reasonably and effectively present, interpret, and represent tolerance semantics. It requires constructing three different kinds of models: (1) presentation model, (2) interpretation model, and (3) representation model.

Presentation model mainly aims at presenting different types of tolerances in a unified, human-readable, and human-understandable way. Representative examples of this kind of model are the presentation models in tolerancing standards (e.g., ISO 1101-2012 [2], ASME Y14.5-2009 [3]) and $\mathrm{CAD} / \mathrm{CAM}$ systems. Presentation model exists mostly in the form of drawing indication, whose meaning can only be read and understood by domain experts and cannot be directly read and understood by computers. This is not enough for computer-aided tolerancing (CAT) in the true sense. True CAT requires tolerance semantics be explicitly represented 
in a computer-readable and computer-understandable form and automatically exchanged among heterogeneous CAD/ CAM systems. To make true CAT possible, the meaning should be firstly interpreted in an unambiguous and rigorous way and then the interpretation is required to be represented in another computer-readable and computer-understandable way.

Interpretation model is used to interpret the meaning of presentation model in an unambiguous and rigorous way. During the past three decades, the construction of an interpretation model of tolerance semantics has gained importance and popularity. Various interpretation models have been presented in this period, where representative examples are parametric model $[4,5]$, offset zone model [6, 7], variational surface model [8], kinematic model [9, 10], degrees of freedom model [11-13], and T-Maps model [14-16]. Interpretation model can be seen as an intermediate model between presentation and representation models. It exists usually in the form of mathematical expression, which also cannot be directly read and understood by computers.

Representation model is constructed to represent interpretation model in an explicit, computer-readable, and computerunderstandable way. Currently, the most widely used representation model of tolerances in industry is the standard for the exchange of product model data (STEP) EXPRESS model [17-19]. This model uses the EXPRESS modeling language to represent tolerance information. Even though using EXPRESS can construct syntactically correct tolerance representation model, it is not capable of representing tolerance semantics explicitly [20]. For this reason, tolerance semantics are not really computer-understandable in STEP EXPRESS model [21]. How to represent the semantics of tolerances to make them computer-understandable remains a challenging task in CAT.

To tackle such challenge, we have introduced the technology of description logic (DL) ontology in the field of the Semantic Web into tolerance semantic representation and presented a DL ontology-based approach to represent the semantics of the type [22], resultant tolerance zone [23], and variational geometry [24] of a single tolerance. As the benefits of DL ontology, such semantics were represented explicitly and expected to be exchanged automatically among heterogeneous CAD/CAM systems. In practice, tolerance modeling not only needs to consider the representation of single tolerance semantics, but also needs to take the representation of composite tolerance semantics into account [25]. To this end, we currently continue the line of research in [22-24] and propose a DL ontology-based approach to represent the semantics of composite tolerances. The present paper takes the representation of the semantics of the composite positional tolerance (CPT) for patterns of holes (POHs) as an example to illustrate the proposed approach. This representation firstly uses DL [26] and Horn rules [27] to represent the structure, constraint, and individual knowledge of the CPT for POHs. Then, it is implemented by web ontology language (OWL) [28] and semantic web rule language (SWRL) [29] and a CPT ontology is obtained through this implementation. Because of the logicbased semantics of OWL and SWRL, this ontology is capable of explicitly representing the semantics of the CPT for POHs. So, it can provide an explicit, computer-readable, and computer-understandable model of CPT. As three advantages of the ontology, consistency checking, knowledge reasoning, and semantic interoperability of the CPT information for POHs can be automatically performed.

The remainder of the paper is organized as follows. An overview of related work is carried out in Section 2. The details of the representation are explained in Section 3. Section 4 reports a prototype implementation of the representation and presents an engineering example to illustrate the advantages of the implemented representation. Section 5 ends the paper with a conclusion.

\section{Related work}

CAT in the true sense requires tolerance semantics be explicitly represented in a both computer-readable and computerunderstandable form and automatically exchanged among heterogeneous $\mathrm{CAD} / \mathrm{CAM}$ systems. Aiming at this requirement, many international and national standards for tolerance information representation and exchange have been successively developed during the past few decades [30]. Among these standards, the most influential and widely applied one is the STEP standard system [31], in which the application protocols (APs) 203 [17], 214 [18], and 242 [19] are broadly accepted and used by commercial CAD/CAM systems. The tolerance information representation language used in these application protocols is EXPRESS [32]. Even though using EXPRESS can construct syntactically correct tolerance information representation model, it cannot represent the semantics of different types of tolerances explicitly because EXPRESS is not based on formal semantics [20]. Consequently, tolerance semantics are just implicitly represented and not really computer-understandable in EXPRESS model [21]. How to represent the detailed semantics of tolerance information in a computer-understandable form has been one of the most popular problems concerned within the industry and the academia in recent years.

In response to this problem, a number of researchers proposed to leverage other existing or develop new knowledge representation languages to construct their respective tolerance representation models. These constructed models can be classified into the following five categories on the basis of the knowledge representation languages used in them: 
- Unified modeling language (UML) model. Rachuri et al. [33] used UML to construct an object-oriented assembly model called "open assembly model" (OAM) to enhance the assembly information content in STEP EXPRESS model. OAM is capable of providing a way for tolerance representation and propagation at the system level and enables plug-and-play with various applications throughout product life cycle (e.g., engineering analysis, virtual assembly, process planning). But it is not computerunderstandable since UML is not based on formal semantics. In addition, there is yet no evidence that OAM includes the representation of the detailed semantics (i.e., resultant tolerance zones and variational geometries) of different types of tolerances.

- Extensive markup language (XML) model. Zhao et al. [34] presented a geometric tolerance representation model by abstracting the explanations and illustrations from ASME geometric tolerancing standards. They further transformed the model into XML schema that can be used to generate XML instance file to satisfy the requirements of geometric tolerance representation in integrated measurement processes. The transformed model can act as an adapter for the communication of geometric tolerance information via the Internet among different application domains. However, it is inappropriate to be used for knowledge reasoning and deduction because XML is only a markup language that has no mathematical basis.

- GeoSpelling formal language model. To express the semantics of the geometrical product specifications (GPS) throughout product life cycle, Dantan et al. [35] firstly proposed a model for GPS whose name is GeoSpelling. They then developed a formal language for the GeoSpelling model [36]. The syntax of this formal language is based on the functions, conditions, and loops in programming language, where functions stand for the declaration of operations and loops, conditions mean the selection of features from a set, and loops correspond to manage a set of features. By this means, the formal language can be applied to express the semantics of the GPS specifications in simulate metrology and assembly or manufacturing sequence. Such applications were planned in their future work.

- Categorical language model. To solve the ambiguous problem caused by describing GPS specifications in natural language in tolerancing standards and technical handbooks, Lu et al. [37] used category language to construct a representation model of the specifications and verification for geometrical tolerances in the framework of the next generation GPS. The representation model was then used by Xu et al. [38] and Qi et al. [39] to develop a knowledgebased system for the manipulation of complicated GPS information. This system provides unambiguous GPS information for designers and metrologists and enables metrology-assisted design and manufacturing to become reality.

- Web ontology language (OWL) model. Fiorentini et al. [40] presented an OWL version of the previous UML model OAM [33] to make it really computer-understandable. They also extended the model to incorporate the reasoning capabilities based on OWL DL and SWRL. This OWL version of OAM can help in achieving various levels of interoperability of tolerance information as required to enable the full potential of product lifecycle management. However, it does not contain the representation of the detailed semantics of different types of tolerances since such representation is not contained in OAM. In response to this limitation, we proposed a DL ontologybased approach to represent the detailed semantics of different types of single tolerances [22-24]. As the advantages of DL ontology, such semantics can be represented explicitly and knowledge reasoning on the resultant representation model can be performed automatically. These advantages are helpful to improve the interoperability of single-tolerance information among heterogeneous applications throughout product life cycle [41]. However, the representation of the semantics of composite tolerances is not included in the proposed approach.

As can be seen from the above literature review, studies about the computerized representation of tolerance information have been paid much attention during the past decade. A number of kinds of tolerance representation models have been presented in this area, where the OWL model is one of the most representative kinds. Since this kind of model did not contain the representation of composite tolerance semantics, we extend it through leveraging DL [26] and Horn rules [27] to model such semantics and using OWL DL [28] and SWRL [29] to implement the model. In this paper, the representation of the semantics of the CPT for POHs is used as an example to illustrate this extension. The main contribution of the paper can be briefly summarized as: The paper proposes a DL ontology-based approach to explicitly represent the semantics of the CPT for POHs. In this approach, a CPT ontology is constructed and developed, which provides a semantic enrichment model of CPT information for the real integration of such information and CAD/CAM systems. Consistency checking, knowledge reasoning, and semantic interoperability of CPT information can be automatically performed. This will ground for the further implementation of CAT in the true sense.

\section{Semantic representation approach}

This section describes an approach to represent the semantics of the CPT for POHs. The schematic representation of this 


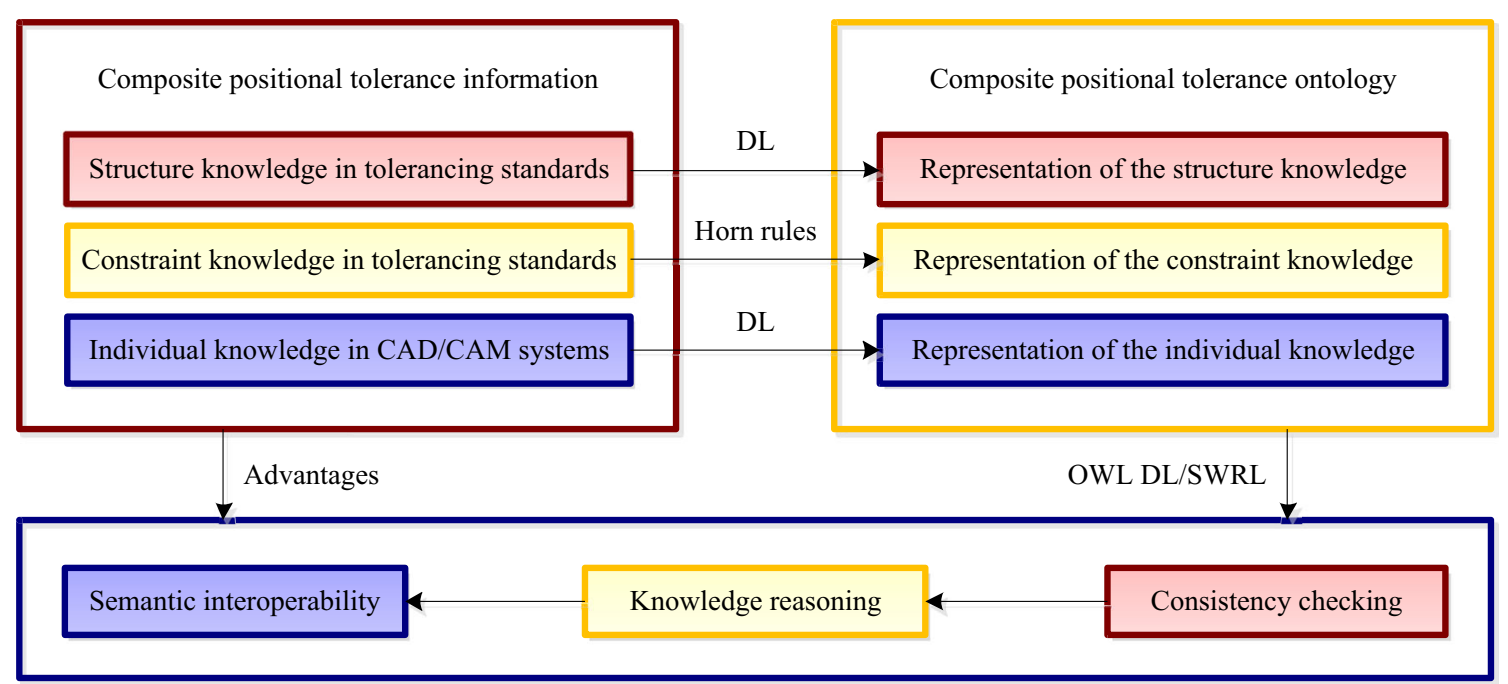

Fig. 1 Schematic representation of the semantic representation approach

approach is shown in Fig. 1. The first step is to leverage DL terminological axioms to represent the structure knowledge of the CPT for POHs in tolerancing standards. Then, a set of Horn rules are designed to express the constraint knowledge that cannot be expressed solely by DL in the second step. The last step is to use DL assertional axioms to describe the individual knowledge of the CPT for POHs extracted from $\mathrm{CAD} /$ CAM systems. Through these three steps, an ontology for the $\mathrm{CPT}$ for POHs can be constructed. Due to the rigorous logicbased semantics of DL and Horn rules, the semantics of the CPT for POHs are explicitly represented and greatly enriched in this ontology. Three advantages of this ontology, i.e., consistency checking, knowledge reasoning, and semantic interoperability of CPT information, which are not currently available in commercial CAD/CAM systems, can be performed automatically. The details of the three steps and the three advantages are respectively explained in the following sub-sections: (1) the representation of the structure knowledge; (2) the representation of the constraint knowledge; (3) the representation of the individual knowledge; and (4) the advantages of the approach.

\subsection{Representation of the structure knowledge}

Structure knowledge in a domain mainly consists of the DL definitions of the terminologies in this domain. To represent the structure knowledge of the CPT for POHs in tolerancing standards, the related terminologies and their definitions should be firstly identified.

In the tolerancing standard ASME Y14.5-2009 [3], there are two types of POHs, where one type is rectangular pattern of holes and the other type is circular pattern of holes. Both of these types of POHs can be imposed a CPT. For example, a rectangular pattern of holes imposed a CPT and a circular pattern of holes imposed a CPT are, respectively, shown in
Figs. 2 and 3. The resultant tolerance zones of these two CPTs are depicted in Figs. 4 and 5, respectively. As can be seen from Figs. 2 and 3, a feature control frame for CPT has one positional tolerance symbol that is applicable to two horizontal segments. The upper segment builds a pattern-locating tolerance zone framework (PLTZF) (e.g., the tolerance zone framework containing $C_{1,1}, C_{1,2}, \ldots, C_{1,6}$ in Fig. 4) which governs the relationship between datum features and pattern. The lower segment builds a feature-relating tolerance zone framework (FRTZF) (e.g., the tolerance zone framework containing $C_{2,1}, C_{2,2}, \ldots, C_{2,6}$ in Fig. 4) that is a refinement of the PLTZF and governs the relationship between features. The resultant tolerance zone of each CPT is the intersection of its PLTZF and FRTZF (e.g., the intersection of the PLTZF $C_{1,1^{-}}$ $C_{1,2^{-}} C_{1,3^{-}} C_{1,4^{-}} C_{1,5^{-}} C_{1,6}$ in Fig. 4 and the FRTZF $C_{2,1^{-}} C_{2,2^{-}}$ $C_{2,3^{-}} C_{2,4}-C_{2,5^{-}} C_{2,6}$ in Fig. 4). To satisfy the requirement of the CPT, its variational geometry must lie in its resultant tolerance zone.

As can be seen from Figs. 4 and 5, a maximum material condition (MMC) is applied to each of the toleranced features $C_{1,1}, C_{1,2}, \ldots, C_{1,6}, C_{2,1}, C_{2,2}, \ldots, C_{2,6}$. This means that the dimensional tolerance and location tolerance (i.e., the positional tolerance of the axis) of each of these holes should meet the maximum material requirement. Taking the MMC applied to $C_{1,1}$ in Fig. 4 as an example, the semantics of such maximum material requirement can be informally described as follows: (1) When the hole $C_{1,1}$ is at MMC (see Fig. 6), the external function size of the actual hole must be greater than or equal to the maximum material virtual size $\phi\left(t-l-t_{1}\right)$. The theoretical position of the axis of the hole is determined by the datum features $A, B$, and $C$ and the theoretically exact sizes $d_{1}$, $d_{2}$, and $d_{3}$. When everywhere of the local actual size of the hole is the maximum material size $\phi(t-l)$, the maximum allowable value of the positional error of the axis is $\phi t_{1}$. (2) When the hole $C_{1,1}$ is not at MMC (i.e., the actual size of the 
Fig. 2 A CPT imposed on a rectangular pattern of holes

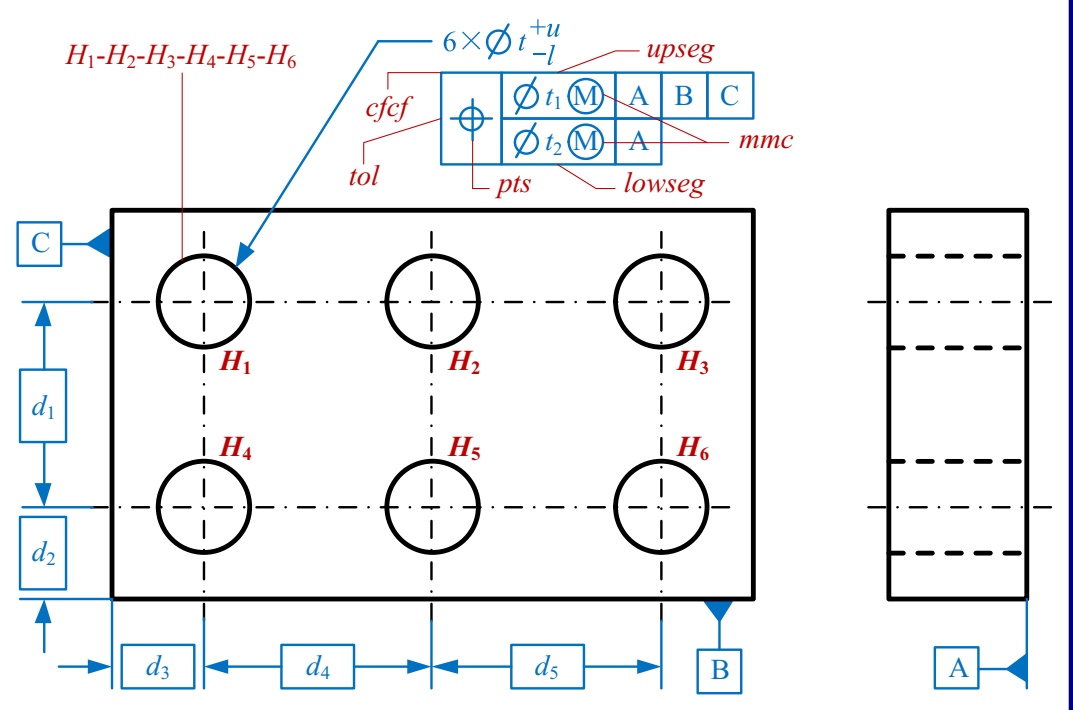

hole $C_{1,1}$ deviates from the maximum material size $\phi(t-l)$ ), the positional error of the axis can be greater than $\phi t_{1}$. As an example, when everywhere of the local actual size of the hole is the minimum material size $\phi(t+u)$, the maximum allowable value of the positional error of the axis is $\phi\left(t_{1}+u+l\right)$. (3) The local actual size of the hole $C_{1,1}$ must lie between $\phi(t-l)$ and $\phi(t+u)$.

In Figs. 2 and 3, all of the MMCs are assigned on toleranced features. In practice, MMC may be applied to size datum features. For example, Fig. 7 shows a MMC assigned on the size datum feature B in a CPT imposed on a circular pattern of holes. This MMC means that the dimensional tolerance of the shaft should meet the maximum material requirement. The semantics of this requirement is informally described as follows: (1) The external function size of the actual shaft must be smaller than or equal to the maximum material virtual size $\phi\left(d_{1}+u_{1}\right)$ and perpendicular to the datum plane $A$. (2) The local actual size of the shaft must lie between $\phi\left(d_{1}-l_{1}\right)$ and $\phi\left(d_{1}+u_{1}\right)$.

According to the above description, the major terminologies used to define the CPT for POHs are composite positional tolerance, pattern of holes, rectangular pattern of holes, circular pattern of holes, resultant tolerance zones, feature control frame, segments, upper segment, PLTZF, lower segment, and FRTZF. All of these terminologies are required to be defined by DL terminological axioms [26]. For example, the
Fig. 3 A CPT imposed on a circular pattern of holes

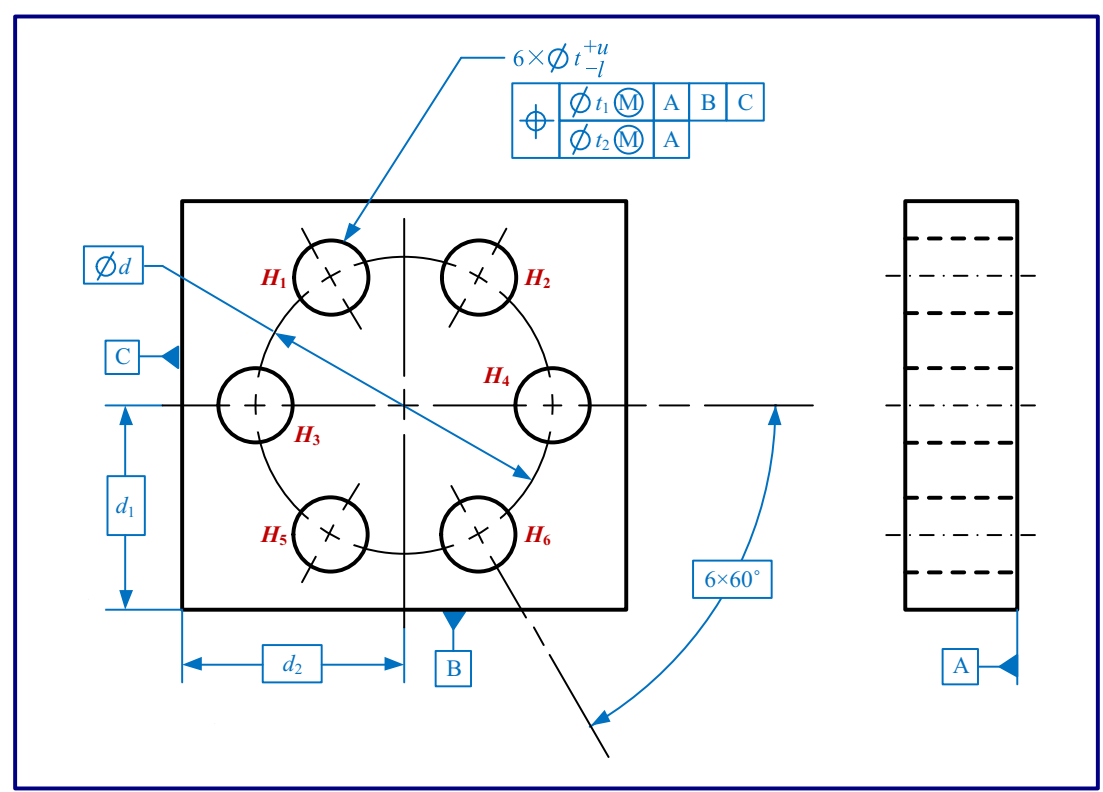


terminology composite positional tolerance can be defined by the following DL terminological axiom:

CompositePositionalTolerance $\equiv$ Tolerance

$\sqcap$ ヨhasTolerancedFeature.PatternOfHoles

$\sqcap \exists$ hasFeatureControlFrame.

CompositeFeatureControlFrame

$\sqcap \exists$ hasResultantToleranceZone.

CompositeToleranceZone

$п$ ヨhas VariationalGeometry.

CompositeVariationalGeometry

where Tolerance is an atomic concept, CompositePositionalTolerance, PatternOfHoles, CompositeFeatureControlFrame, CompositeToleranceZone, and CompositeVariationalGeometry are all complex concepts. The DL definitions of PatternOfHoles, CompositeFeatureControlFrame, and CompositeToleranceZone are listed in Appendix A (for the DL definition of CompositeVariationalGeometry, please refer to [24]).

Now a model-theoretic semantics can be assigned to the axiom defining the terminology composite positional tolerance to make it computer-understandable. This semantics is expressed using the notion of an interpretation $I=\left(\Delta^{I},{ }^{I}\right)$, where $\Delta^{I}$ is a universal set of domain individuals and ${ }^{I}$ is an interpretation function which interprets each concept $C$ to a subset $C^{I}$ of $\Delta^{I}$ (e.g., CompositePositionalTolerance ${ }^{I} \subseteq \Delta^{I}$ ), each role $R$ to a subset $R^{I}$ of $\Delta^{I} \times \Delta^{I}$ (e.g., hasTolerancedFeature $^{I} \subseteq \Delta^{I} \times \Delta^{I}$ ), each conjunction of concepts $C \sqcap D$ to a set $C^{I} \cap D^{I}$ (e.g., (Tolerance $\sqcap \exists$ hasTolerancedFeature.PatternOfHoles $)^{I}=$ Tolerance $^{I} \cap($ hasTolerancedFeature.PatternOfHoles $)^{I}$ ), each disjunction of concepts $C \sqcup D$ to a set $C^{I} \cup D^{I}$ (e.g., (UpperSegment $\sqcup$ LowerSegment) ${ }^{I}=$ UpperSegment ${ }^{I} \cup$ LowerSegment $t^{I}$ ), each existence restriction $\exists R$. $C$ to a set $\left\{x \in \Delta^{I} \mid \exists y\right.$. $\left.(x, y) \in R^{I} \wedge y \in C^{I}\right\}$ (e.g., ( $\exists$ has-

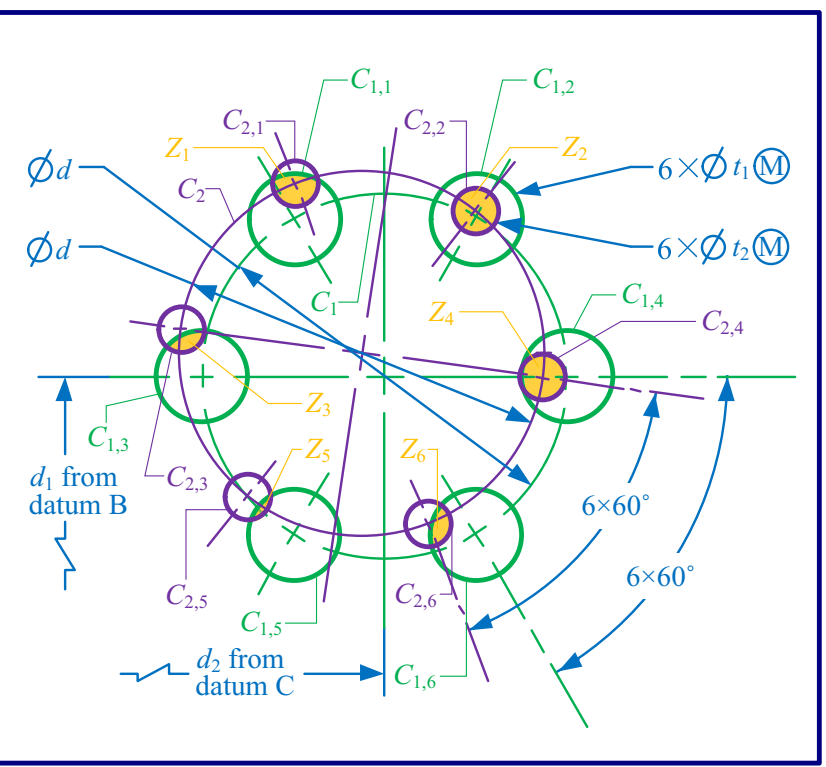

Fig. 5 One possible resultant tolerance zone of the CPT in Fig. 3

TolerancedFeature.PatternOfHoles $)^{I}=\left\{x \in \Delta^{I} \mid \exists y .(x, y) \in\right.$ hasTolerancedFeature $^{I} \wedge y \in$ PatternOfHoles $\left.\left.^{I}\right\}\right)$, and each data existence restriction $\exists R_{\mathrm{d}}$.d to a set $\left\{x \in \Delta^{I} \mid \exists y .(x, y) \in R_{\mathrm{d}}^{I} \wedge\right.$ $y \in \mathrm{d}\}$ (e.g., ( $\exists$ hasToleranceValue.float $)^{I}=\left\{x \in \Delta^{I} \mid \exists y .(x\right.$, y) $\in$ hasToleranceValue ${ }^{I} \wedge y \in$ float $\}$ ).

With this interpretation function, the axiom is explicitly interpreted as: An individual $x$ is said to be a CPT, if and only if (1) $x$ is a tolerance; (2) there exist a pattern of holes $y_{1}$ such that $y_{1}$ is the toleranced features of $x$; (3) there exist a composite feature control frame $y_{2}$ such that $y_{2}$ is the feature control frame of $x$; (4) there exist a composite tolerance zone $y_{3}$ such that $y_{3}$ is the resultant tolerance zone of $x$; and (5) there
Fig. 4 One possible resultant tolerance zone of the CPT in Fig. 2

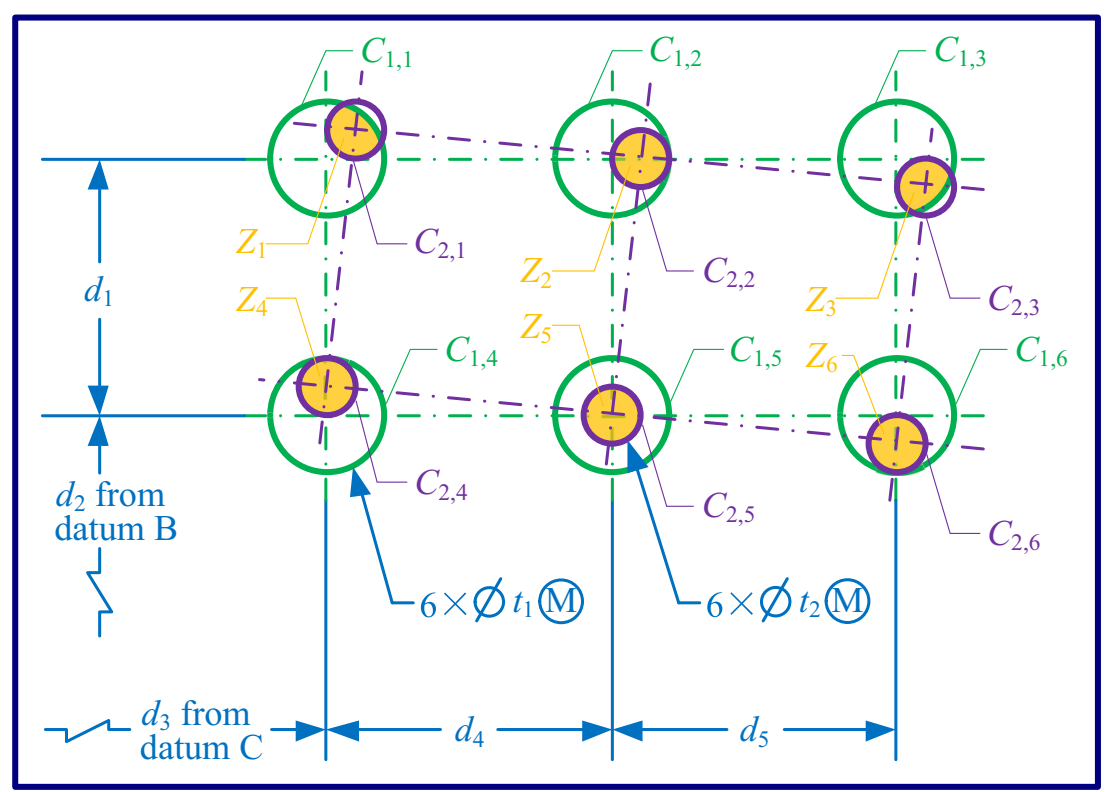


Fig. 6 The semantics of the MMC assigned on the toleranced feature $C_{1,1}$ in Fig. 4
Tolerance zone when $C_{1,1}$ is at $\mathrm{MMC}$

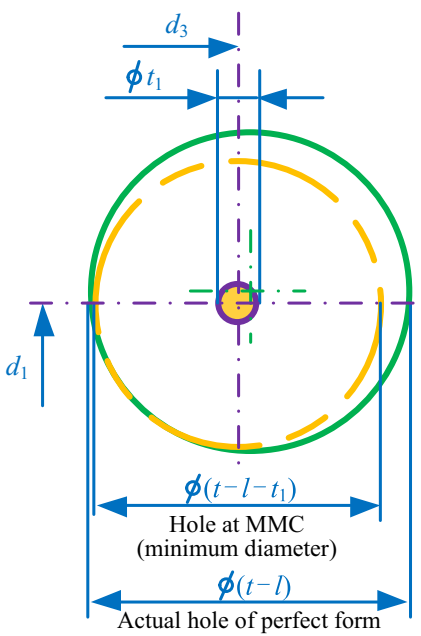

Tolerance zone when $C_{1,1}$ is not at $\mathrm{MMC}$

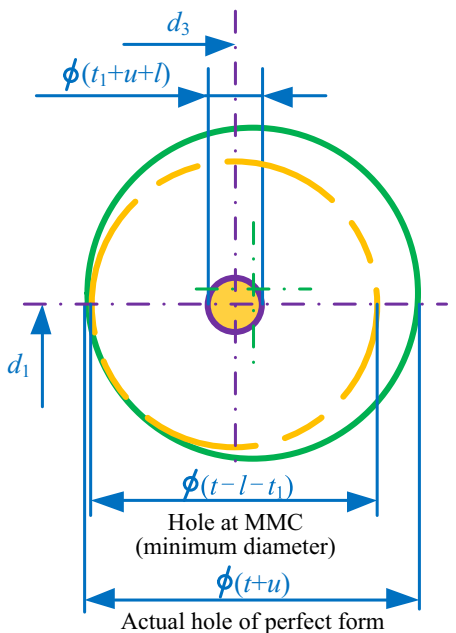

exists a composite variational geometry $y_{4}$ such that $y_{4}$ is the variational geometry of $x$. Such interpretation is easily understood by both humans and computers. Therefore, the representation of the definition of the terminology composite positional tolerance has directly computer-understandable semantics. Correspondingly, the model-theoretic semantics can also be assigned to other axioms in the expressions in Appendix A and the semantics of these axioms can also be interpreted in a similar way.

As can be summarized from the above example (i.e., the representation of the semantics of the terminology composite positional tolerance), the semantics of the structure knowledge of the CPT for POHs can be represented through the following steps: (1) Identify the related terminologies and their definitions from the tolerancing standard ASME Y14.5-2009. (2) Specify the atomic concepts, complex concepts, object roles, and data roles according to the identified terminologies. (3) Leverage DL terminological axioms to define complex concepts. (4) Assign a model-theoretic semantics to each specified atomic concept, each specified object role, each specified data role, and each defined complex concept.

\subsection{Representation of the constraint knowledge}

Constraint knowledge in a domain can be seen as some computer-understandable rules that describe the constraint
Fig. 7 A MMC assigned on the size datum feature $B$

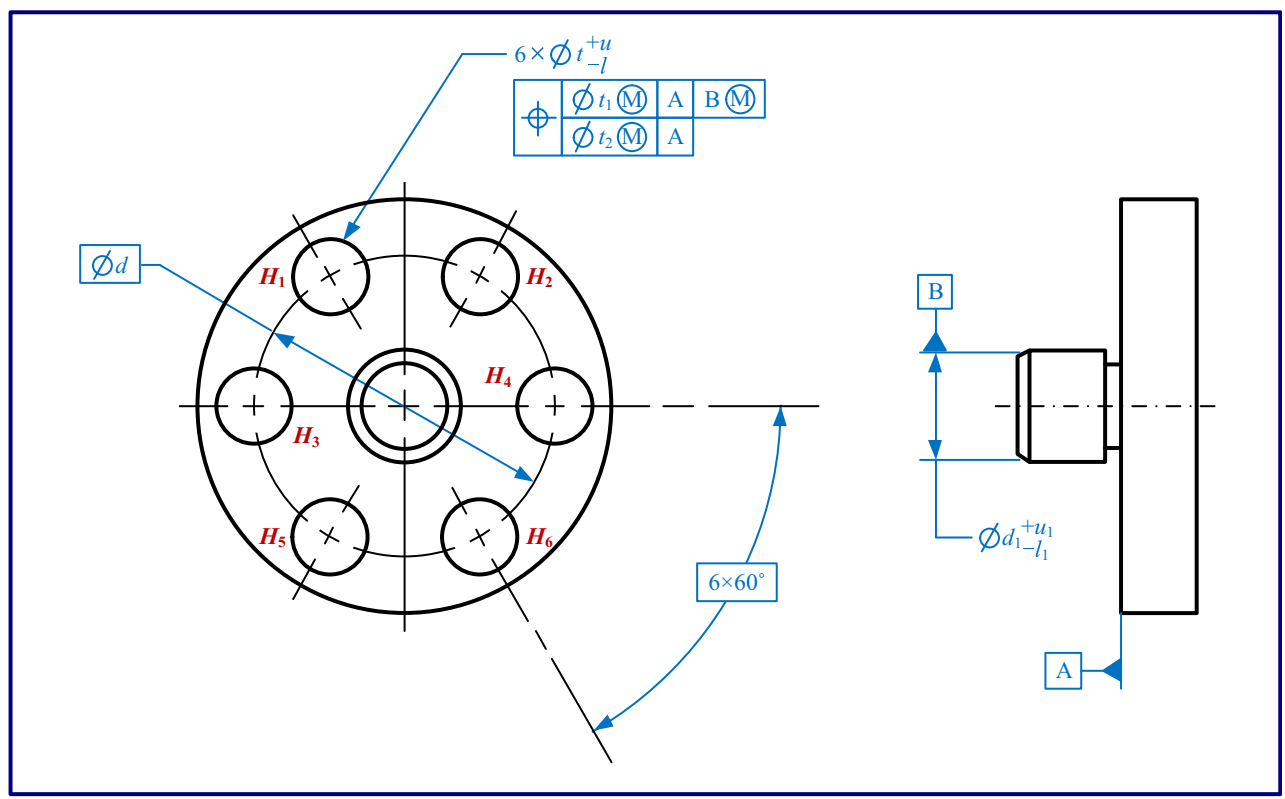


Fig. 8 A component with a rectangular pattern of holes imposed a CPT

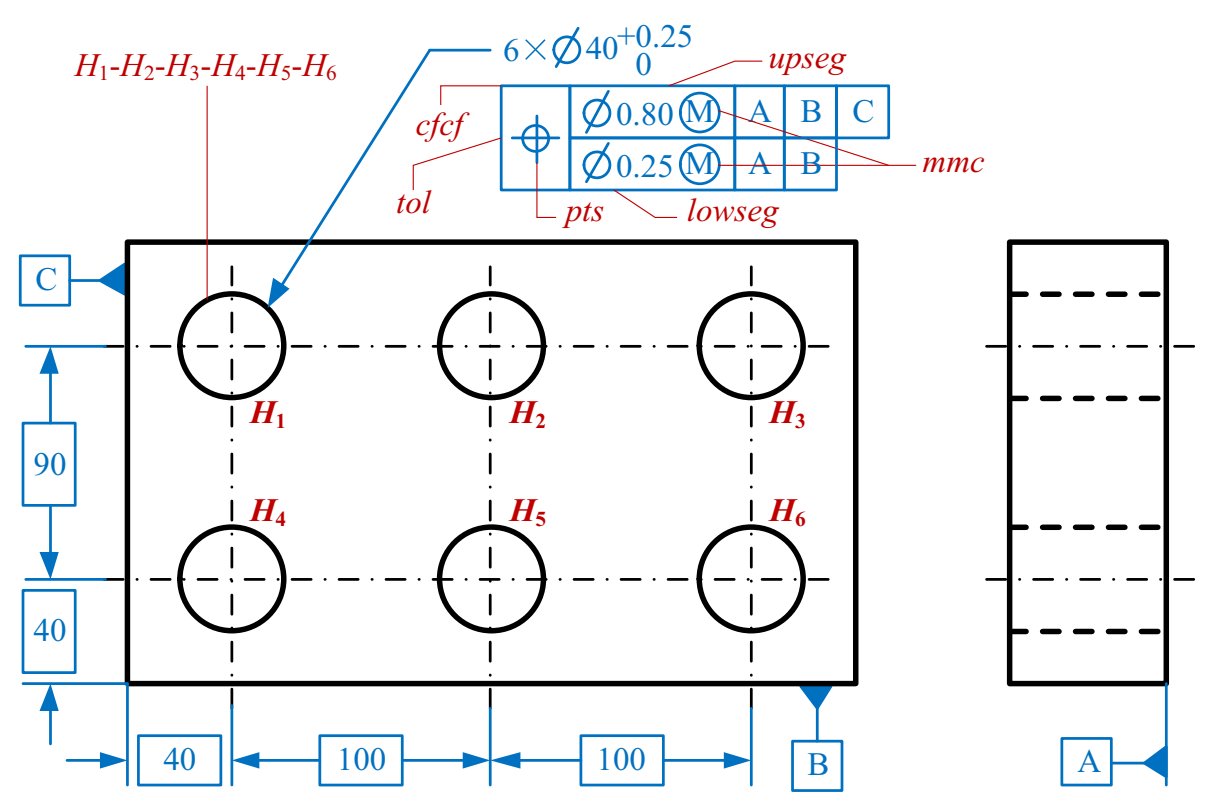

relationships among the objects (that correspond to concepts in DL) and relations (that correspond to roles in DL) in this domain. To represent the constraint knowledge of the CPT for POHs in tolerancing standards, the first step is to determine the constraint relationships which require to be described.

From Figs. 2, 4, and 6 (or Figs. 3, 5, and 6), the semantics of a CPT can be understood as: the variational geometry of this CPT must fall inside the intersection of its PLTZF and FRTZF. This semantics implies three aspects of constraint relationships of the CPT: (1) the constraint relationships between its upper segment and its PLTZF; (2) the constraint relationships between its lower segment and its FRTZF; and (3) the constraint relationships between its variational geometry and the intersection of its PLTZF and FRTZF.

For the first aspect of constraint relationships, the tolerance value $t_{1}$ in the upper segment in Fig. 2 (or Fig. 3 ) is the diameter of each of the six cylindrical tolerance zones in the PLTZF in Fig. 4 (or Fig. 5). The symbol $\mathbb{M}$ means that $t_{1}$ meets the MMC. This meaning implies the constraint relations that have been depicted in Fig. 6. According to Horn logic [27], these constraint relationships can be described by the Horn rule hasMeetMaximumMaterialCondition $\left(t_{1}, m m c\right)$ in Appendix
Fig. 9 One possible resultant tolerance zone of the CPT in Fig. 8

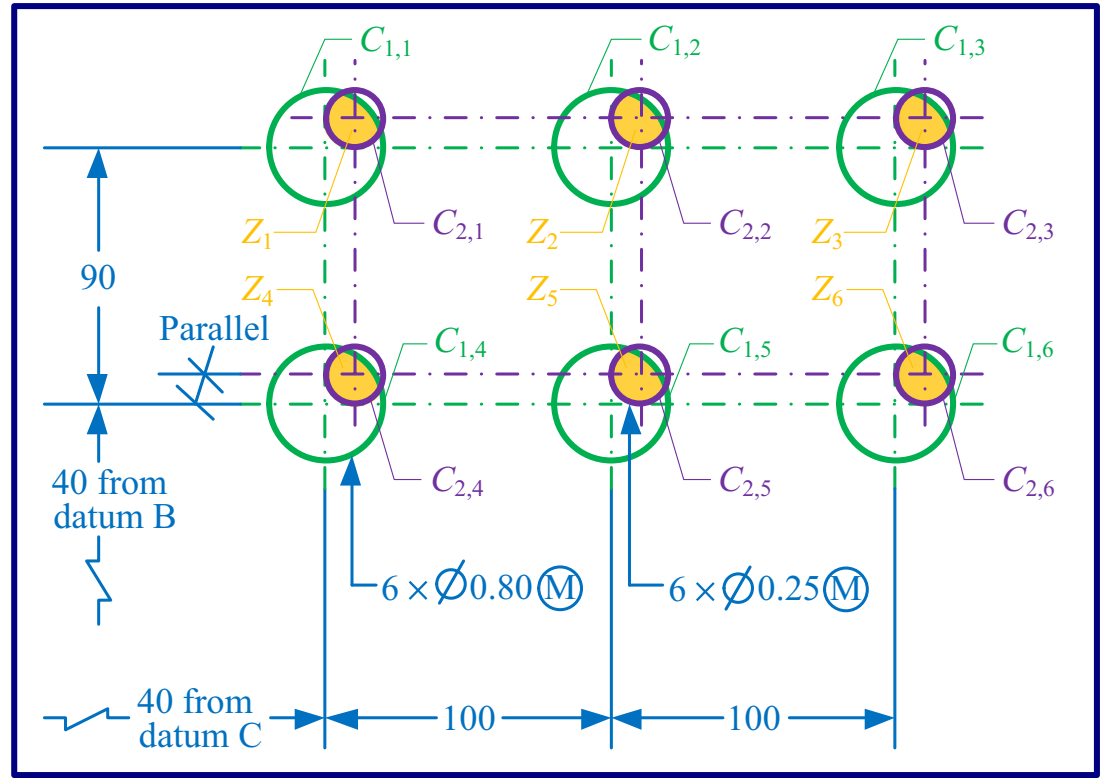


Fig. 10 The semantics of the MMC assigned on $C_{1, i}(i=1,2$, ..., 6) in Fig. 9
Tolerance zone when $C_{1, i}$ is at $\mathrm{MMC}$

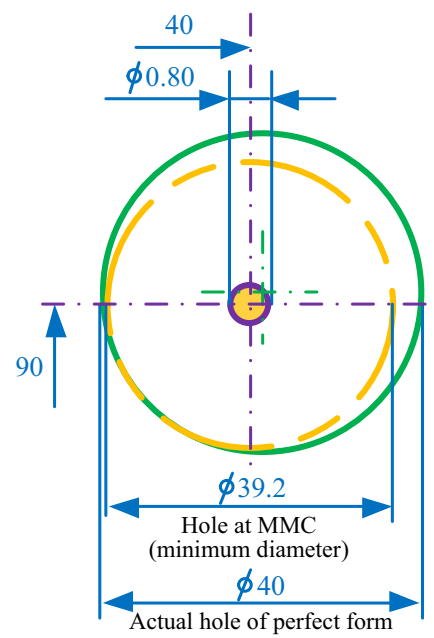

Tolerance zone when $C_{1, i}$ is not at MMC

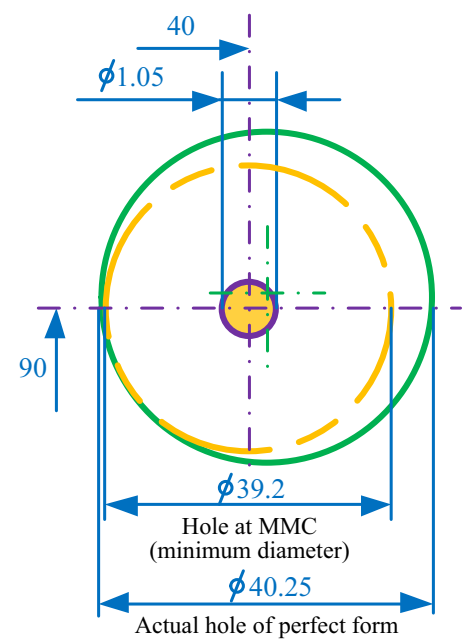

B. Similarly, the letter $A$ is used to orient the six $\phi t_{1}$ cylindrical tolerance zones perpendicular to the datum feature $A$. The letters $B$ and $C$ are used to locate the six $\phi t_{1}$ cylindrical tolerance zones with fundamental dimensions to the datum features $B$ and $C$. According to Horn logic [27], these constraint relationships are described by the Horn rule isPLTZFOf $\left(x_{4}, x_{1}\right)$ in Appendix B. Now a model-theoretic semantics can be assigned to this Horn rule to make it computer-understandable. This semantics is also described using the interpretation $I=\left(\Delta^{I},{ }^{I}\right)$. The description is: CompositePositionalTolerance ${ }^{I}$ $\cap$ CompositeFeatureControlFrame $^{I} \cap \ldots \cap$ hasValue $^{I} \subseteq$ isPLTZFOf ${ }^{I}$. This description can also be directly understood by computers. So the described constraint relationships also have directly computer-understandable semantics.

For the second aspect of constraint relationships, the tolerance value $t_{2}$ in the lower segment in Fig. 2 (or Fig. 3) is the diameter of each of the six cylindrical tolerance zones in the FRTZF in Fig. 4 (or Fig. 5). The symbol (M) means $t_{2}$ meets the MMC. The FRTZF controls the location of the six $\phi t_{2}$ cylindrical tolerance zones with fundamental dimensions to each other. It is free to translate and rotate within the boundaries of the PLTZF. The letter A is used to orient the six $\phi t_{2}$ cylindrical tolerance zones perpendicular to the datum feature A. These constraint relationships can also be described by a Horn rule

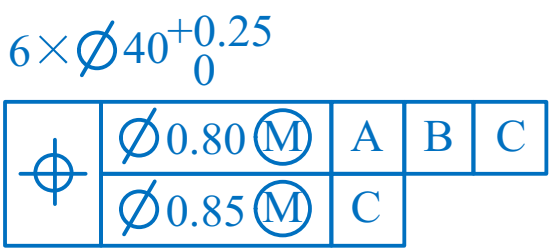

Fig. 11 One inconsistent drawing indication of CPT
isFRTZFOf that is similar to the Horn rule isPLTZFOf $\left(x_{4}, x_{1}\right)$ in Appendix B.

For the third aspect of constraint relationships, the variational geometry of the CPT in Fig. 2 (or Fig. 3) must fall inside the intersection of its PLTZF and FRTZF in Fig. 4 (or Fig. 5). This constraint relationship can be described by the Horn rule MeetRequirementOfCPT $\left(x_{2}, x_{1}\right)$ in Appendix B. Like the Horn rule isPLTZFOf $\left(x_{4}, x_{1}\right)$ in Appendix B, a model-theoretic semantics can also be assigned to this Horn rule to make it computer-understandable.

If a CPT specified in the way shown in Fig. 7 (i.e., in the upper or lower segment, a MMC is assigned on the size datum features), the three aspects of the constraint relationships for this figure can be described and the model-theoretic semantics can be assigned to the descriptions in a similar way.

From the above explanations, the semantics of the constraint knowledge of CPT for POHs can be represented through determining the constraint relationships that require to be described, using Horn rules to describe these constraint relationships, and assigning a model-theoretic semantics to each Horn rule.

\subsection{Representation of the individual knowledge}

Individual knowledge in a domain can be seen as some computer-understandable statements of the concrete individuals in this domain. To represent the individual knowledge of the CPT for POHs in CAD/CAM systems, the first step is to extract the concrete individuals in this CPT.

Using the application program interface (API) of a CAD/ CAM system, the concrete individuals in CPTs can be easily extracted from this system. For instance, assume the part in Fig. 2 has been designed in a CAD/CAM system. Using the 


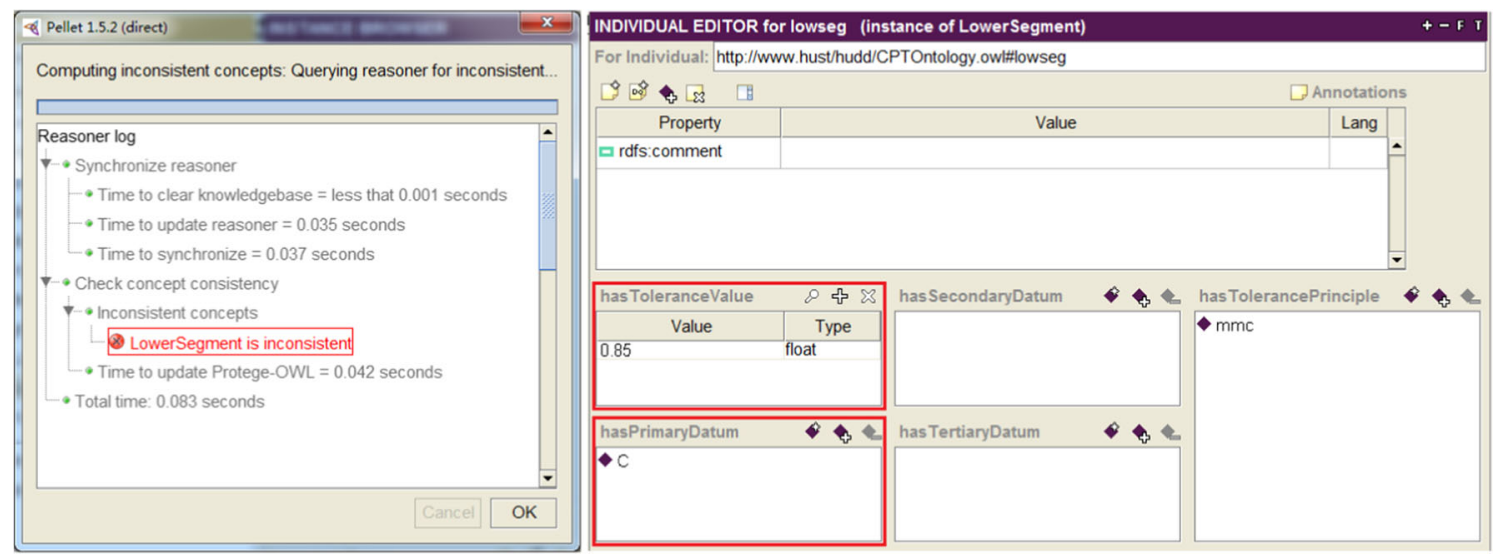

Fig. 12 Checking result of the two inconsistencies in Fig. 11

API of the system, the concrete individuals in the CPT imposed on the rectangular pattern of holes of this part are extracted as follows: (1) tol is a CPT; (2) $\mathrm{H}_{1}-\mathrm{H}_{2}-\mathrm{H}_{3}-\mathrm{H}_{4}-\mathrm{H}_{5}-\mathrm{H}_{6}$ is a rectangular pattern of holes and is the toleranced feature of tol; (3) $c f c f$ is a composite feature control frame and is the feature control frame of tol; (4) pts is a positional tolerance symbol and is the tolerance symbol of $c f c f$; (5) upseg is an upper segment and is a segment of $c f c f$; (6) lowseg is a lower segment and is a segment of $c f c f ;(7) t_{1}$ is the tolerance value in upseg; (8) $m m c$ is the MMC and is the tolerance principle in upseg; (9) $A$ is a datum feature and is the primary datum in upseg; (10) $B$ is a datum feature and is the secondary datum in upseg; (11) $C$ is a datum feature and is the tertiary datum in upseg; (12) $t_{2}$ is the tolerance value in lowseg; (13) $\mathrm{mmc}$ is also the tolerance principle in lowseg; and (14) $A$ is also the primary datum in lowseg.

The second step is to use some computer-understandable statements to describe the extracted concrete individuals. Here DL assertional axioms [26] are used and thus the extracted concrete individuals from (1) to (14) can be described by the assertional axioms in Appendix C. This description is based on model-theoretic semantics. So it can also be directly understood by computers.

In addition to representing the CPT itself, its detailed semantics (i.e., its resultant tolerance zone and variational geometry) can also be represented in this way. Such representation is not available in commercial CAD/CAM systems since most of these systems use STEP AP 203/AP 214/AP 242 to

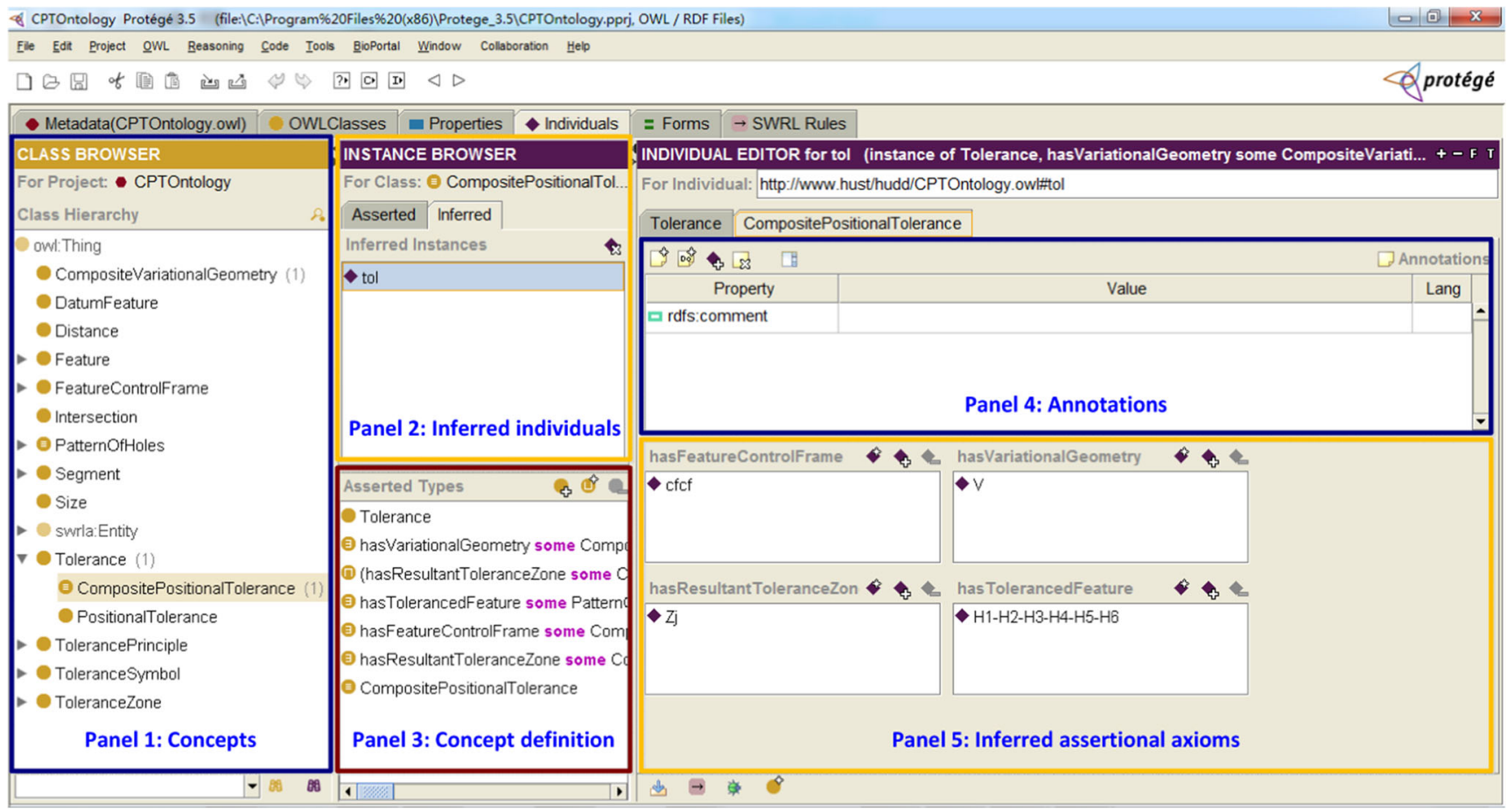

Fig. 13 Inferred result of the example of knowledge reasoning based on OWL DL 
express the tolerance information and these APs will not contain the expression of the detailed semantics of tolerance information [21]. Hence, capturing and representing such detailed semantics is a particular feature of the approach.

To represent the detailed semantics of a CPT, its resultant tolerance zone and variational geometry must be firstly established. This work can be completed through using Liu et al.'s establishment methods [25, 42, 43]. After establishing the resultant tolerance zone and variational geometry, the detailed semantics can also be described by DL assertional axioms [26]. For example, a DL description of the resultant tolerance zone of the CPT in Fig. 2 (i.e., the resultant tolerance zone in Figure 4) is provided in Appendix D.

From the representation process of the individual knowledge in Figs. 2 and 4, the representation process of the individual knowledge of the CPT for POHs is summarized as follows: (1) Use the APIs of CAD/CAM systems to extract the CPT information from these systems. (2) Apply Liu et al.'s establishment methods to establish the resultant tolerance zone and variational geometry of this tolerance. (3) Use DL assertional axioms to describe the extracted tolerance information and the established resultant tolerance zone and variational geometry.

\subsection{Advantages of the approach}

The most prominent feature of DL is that it can provide the maximum expressive power under the prerequisite of ensuring computational completeness and decidability. This enables consistency checking of DL ontology, knowledge reasoning on DL ontology, and semantic interoperability between DL ontologies. In addition, knowledge reasoning on DL ontology can also be combined with Horn rules [44]. Since the approach has leveraged DL and Horn rules to construct a CPT ontology, it mainly has the following advantages:

- Consistency checking of the CPT ontology. Consistency checking of the ontology checks whether there are inconsistencies in the DL definitions of concepts and roles and whether instantiations of concepts and roles would create inconsistencies. Using a DL inference engine, the consistency of the CPT ontology can be checked automatically. Then knowledge reasoning on the ontology can be performed if there is no inconsistency in the ontology.

- Knowledge reasoning on the CPT ontology. Knowledge reasoning on the ontology takes as input the explicit knowledge in CPT representation and returns as output the implicit knowledge in this representation. It mainly uses the inference capability of DL and Horn rules and is performed by an inference engine. The inference engine takes as input the representation of individual knowledge to obtain new conclusions. After performing knowledge reasoning on the CPT ontology, the newly generated knowledge in it will be available for many downstream tasks, where semantic interoperability is one of these tasks.

- Semantic interoperability of the information of CPT. Semantic interoperability of tolerance information aims to exchange the semantics of the tolerance information between heterogeneous $\mathrm{CAD} / \mathrm{CAM}$ systems to overcome the limitation of traditional STEP based exchange method [41]. It can be implemented using the mechanisms of knowledge reasoning and semantic similarity assessment [45], which can determine semantically equivalent concept (role) pairs and semantically similar concept (role) pairs between two tolerance ontologies, respectively.

\section{Prototype implementation and engineering example}

This section firstly reports a prototype implementation of the semantic representation approach. It then presents an engineering example to illustrate the advantages of the approach.

\subsection{Prototype implementation}

The representations of the structure and constraint knowledge are implemented using the OWL DL language [28] and the SWRL language [29] in Protégé 3.5 [46], respectively. The representation of the individual knowledge is implemented by using the OWL DL language, CAD/CAM systems' Java API, Protégé-OWL API, and Java programming language. Both consistency checking of the CPT ontology and knowledge reasoning on the ontology are performed by the Jess inference engine [47].

\subsection{Engineering example}

A component with a rectangular pattern of holes imposed a CPT is taken as an example to illustrate the advantages of the semantic representation approach. This component and the CPT are, as shown in Fig. 8, designed in a CAD/CAM system. Starting from this designed CPT, the working procedure of the approach mainly contains four steps.

The first step is to extract the tolerance information from the CAD/CAM system. The information of the CPT in Fig. 8 is extracted using the APIs of the CAD/CAM system.

The second step is to instantiate the CPT ontology according to the extracted tolerance information. Using ProtégéOWL API, the CPT ontology is instantiated by the extracted CPT information.

The third step is to establish the resultant tolerance zone and variational geometry of the CPT. The resultant tolerance zone and variational geometry of the CPT in Fig. 8 are established by using the establishment methods proposed by 
Fig. 14 A SWRL rule used to simulate the CPT of a pattern of holes

\begin{tabular}{|c|c|}
\hline sWRL Rule & 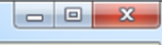 \\
\hline Name Comment & \\
\hline Name & \\
\hline http://www.hust/hudd/CPTOntology.owl\# & \\
\hline SWRL Rule & \\
\hline 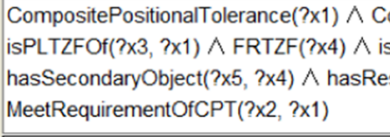 & $\operatorname{PLTZF}(? \times 3) \wedge$ \\
\hline 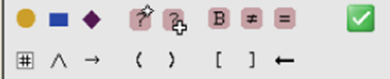 & \\
\hline
\end{tabular}

Liu et al. For instance, one possible resultant tolerance zone of the CPT in Fig. 8 is shown in Fig. 9, where the semantics of the MMC assigned on each of $C_{1,1}, C_{1,2}, \ldots, C_{1,6}$ is depicted in Fig. 10. Taking the MMC applied to $C_{1,1}$ as an example, the semantics can be described as follows: (1) When the hole $C_{1,1}$ is at MMC, the external function size of the actual hole must be greater than or equal to the maximum material virtual size $\phi 39.2$. When everywhere of the local actual size of the hole is the maximum material size $\phi 40$, the maximum allowable value of the positional error of the axis is $\phi 0.80$. (2) When the hole $C_{1,1}$ is not at $\mathrm{MMC}$, the positional error of the axis can be greater than $\phi 0.80$. For instance, when everywhere of the local actual size of the hole is the minimum material size $\phi 40.25$, the maximum allowable value of the positional error of the axis is $\phi 1.05$. (3) The local actual size of the hole $C_{1,1}$ must lie between $\phi 40$ and $\phi 40.25$. The semantics of the MMC applied to each of $C_{1,2}, C_{1,3}, \ldots, C_{1,6}, C_{2,1}, C_{2,2}, \ldots, C_{2,6}$ can be depicted/described in a similar way.

The last step is to instantiate the CPT ontology on the basis of the established resultant tolerance zone and variational geometry. Using Protégé-OWL API, the CPT ontology is further instantiated according to the established resultant tolerance zone and variational geometry.
Now the advantages of the approach are illustrated as follows:

- Consistency checking of the CPT ontology. Consistency checking is a particular mechanism of an OWL DL ontology. Such mechanism cannot be performed on an STEP EXPRESS model. Here is an example of using consistency checking mechanism to check the consistency of the drawing indication of a CPT. Assume a designer designs a CPT depicted in Fig. 11 for the rectangular pattern of holes in Fig. 8. Then, two inconsistent places will be, as shown in Fig. 12, automatically checked out by the consistency checking mechanism in Protégé after extracting the information of this CPT and instantiating the CPT ontology based on the extracted information. The first inconsistent place is in the indication of the tolerance value in lower segment. According to the definition of a CPT [2, 3], the tolerance in lower segment must be tighter than the tolerance value in upper segment (i.e., the tolerance value in lower segment must be smaller than the tolerance value in upper segment). Thus, there is an inconsistency in this place. The second inconsistent place is in the indication of the datum feature in lower segment. From the definition
Fig. 15 Inferred result of the example of knowledge reasoning based on SWRL rules

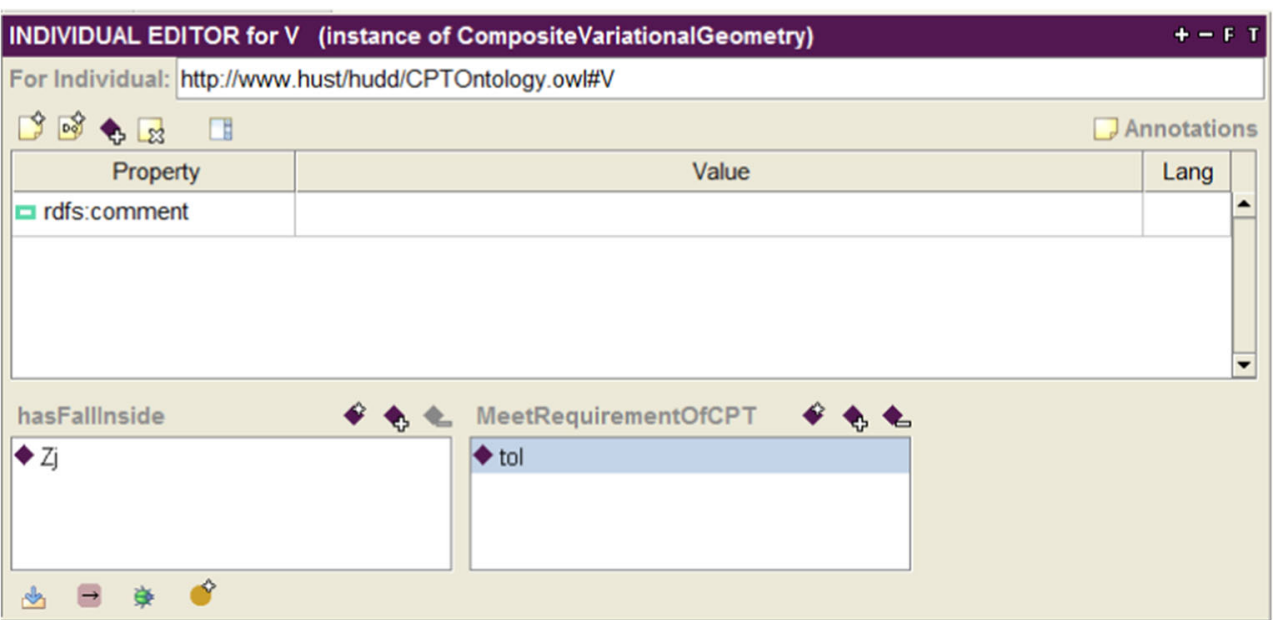




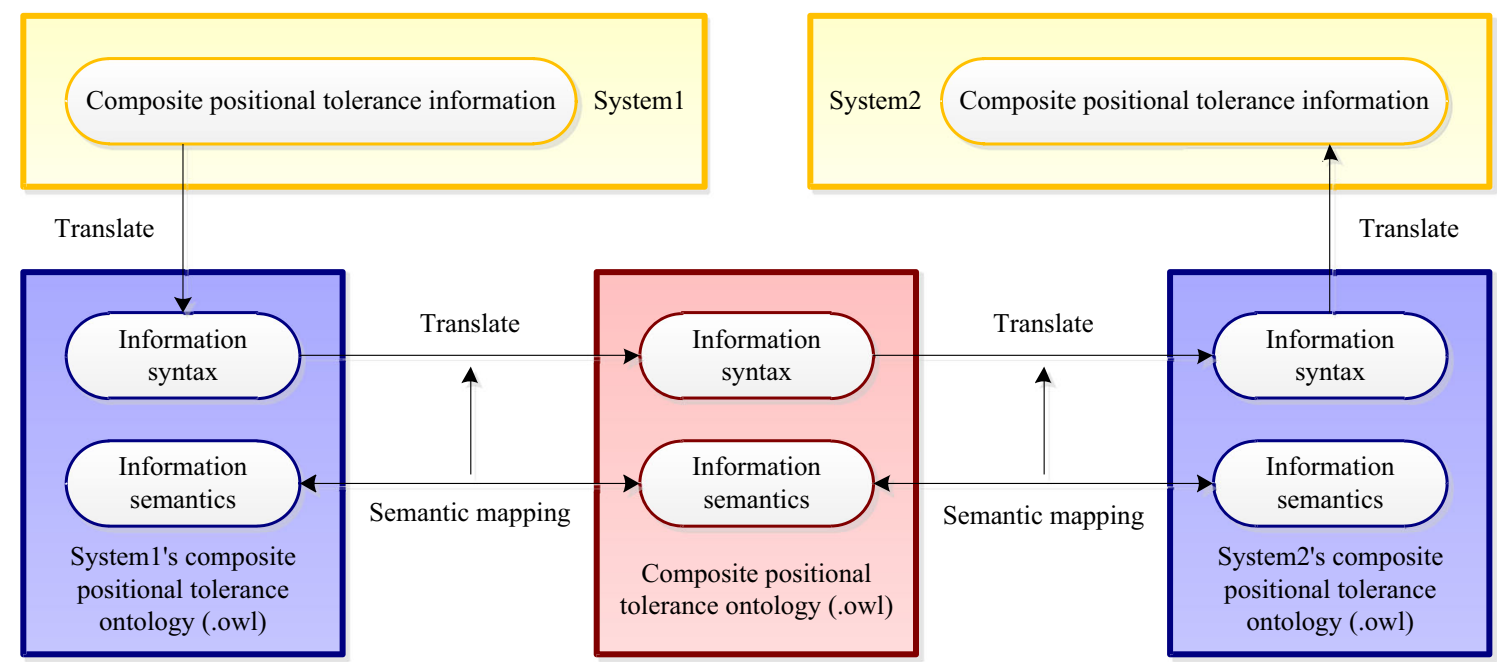

Fig. 16 Semantic interoperability of the information of CPT

of a CPT $[2,3]$ and the definition of a lower segment (see Appendix A), the tertiary datum cannot be firstly repeated in this segment. So, an inconsistency occurs at this place. It should be pointed out that although such checking appears straightforward, current commercial CAD/CAM systems incorporate no such capability yet.

- Knowledge reasoning on the CPT ontology. Knowledge reasoning is a mechanism to generate new knowledge in the CPT ontology. It can be performed if and only if the CPT ontology is checked to be consistent. Knowledge reasoning is carried out using the reasoning capability of OWL DL and SWRL rules. Here are two examples: (1) An example of knowledge reasoning based on OWL DL. Assume tol is a tolerance that has the rectangular pattern of holes $\mathrm{H}_{1}-\mathrm{H}_{2}-\mathrm{H}_{3}$ $H_{4}-H_{5}-H_{6}$ in Fig. 8 as its toleranced feature, has the composite feature control frame $c f c f$ as its feature control frame, has the composite tolerance zone $Z_{j}(j=1,2, \ldots, 6)$ in Fig. 9 as its resultant tolerance zone, and has the composite variational geometry $V$ as its variational geometry. If these statements are taken as input of a DL inference engine, the inference engine will return as output the statements (i.e., inferred assertional axioms) shown in Fig. 13. This figure is commented to show different components of the CPT ontology. It is split into five panels. Panel 1 lists all concepts and their hierarchies in the ontology. In this panel the concept CompositePositionalTolerance is chosen, thus all its asserted and inferred individuals are listed in panel 2 and its asserted definition is shown in panel 3. In panel 2, the inferred individual of CompositePositionalTolerance is shown and selected. So, the annotations and inferred assertional axioms related to this individual are depicted in panels 4 and 5, respectively. According to the inferred result in Fig. 13, the following statements are obtained: tol is a CPT which has the rectangular pattern of holes $\mathrm{H}_{1}-\mathrm{H}_{2}-\mathrm{H}_{3}-\mathrm{H}_{4}-\mathrm{H}_{5}-\mathrm{H}_{6}$ in Fig. 8 as its toleranced feature, has the composite feature control frame $c f c f$ as its feature control frame, has the composite tolerance zone $Z_{j}(j=1,2, \ldots, 6)$ in Fig. 9 as its resultant tolerance zone, and has the composite variational geometry $V$ as its variational geometry. (2) An example of knowledge reasoning based on SWRL rules. The following example shows how to use the SWRL rule in Fig. 14 (corresponds to the Horn rule MeetRequirementOfCPT $\left(x_{2}, x_{1}\right)$ in Appendix B) to conduct CPT simulation of the rectangular pattern of holes in Fig. 8. According to Figs. 8 and 9, the following statements are asserted: $t o l$ is a CPT; $C_{1, j}(j=1,2, \ldots, 6)$ is the PLTZF of $t o l ; C_{2, j}$ is the FRTZF of $t o l$; and the intersection of $C_{1, j}$ and $C_{2, j}$ is the resultant tolerance zone of tol. Assume $V$ is a composite variational geometry generated from a given component with a rectangular pattern of holes. Using the SWRL rule in Fig. 14, whether the rectangular pattern of holes can meet the requirement of the CPT in Fig. 8 can be checked automatically by the Jess inference engine. That is, the rectangular pattern of holes meet the requirement of the CPT if and only if $V$ falls inside the intersection of $C_{1, j}$ and $C_{2, j}$ (see Fig. 15).

- Semantic interoperability of the information of CPT. The developed CPT ontology can be, as shown in Fig. 16, used as a bridge ontology to implement the semantic interoperability of the information of CPT between heterogeneous CAD/CAM systems. Such interoperability process is summarized as follows: The CPT information in System 1 is translated to the information syntax in the System1's CPT ontology (i.e., the individuals in the System1's ontology) and the semantic mappings between the System1's ontology and the developed CPT ontology and between the developed ontology and the System2's CPT ontology are firstly established. Then, the information syntax in the System1's ontology can be translated to the information syntax in the developed 
ontology (i.e., the individuals in the developed ontology) with the help of the established semantic mapping between the System1's ontology and the developed ontology. Analogously, the information syntax in the developed ontology is then translated to the information syntax in the System2's ontology (i.e., the individuals in the System2's ontology) by means of the established semantic mapping between the developed ontology and the System2's ontology. Lastly, the information syntax in the System2's ontology is translated to the CPT information in System2. Since the three ontologies, in which the semantics of CPT information are explicitly and formally interpreted and represented by OWL DL, are properly mapped, the translations of the information syntax between them enable the translations of information semantics as well [48]. Ahmed and Han [41] have used OWL DL ontology to implement the semantic interoperability of tolerance information. Therefore, for the detailed process and concrete example of the semantic interoperability of tolerance information, please refer to their work.

\section{Conclusion}

In this paper, we have continued our research on the recent approach to the representation of the semantics of a single tolerance in [22-24] and proposed a DL ontology-based approach to explicitly representing the semantics of the CPT for POHs. This approach provides a semantic enrichment model of the information of CPT for the real integration of such information and CAD/CAM systems. In detail, we firstly constructed representation models of the structure, constraint, and individual knowledge of the CPT for POHs leveraging DL terminological axioms, Horn rules, and DL assertional axioms, respectively. We then implemented these models using the OWL/SWRL ontologybased technology. A CPT ontology was developed in this implementation. We also presented an engineering example to illustrate the advantages of the proposed approach. As can be seen from the example, the developed ontology has explicitly computer-understandable semantics because of the logic-based semantics of OWL and SWRL. Consistency checking, knowledge reasoning, and semantic interoperability of CPT information can be automatically performed, which will lay a solid basis for the further implementation of CAT in the true sense.

The wider application of the DL ontology based approach for explicitly representing tolerance semantics is mainly limited by the limitations of an OWL/SWRL ontology, which include [49] the following: (1) The expressiveness of the OWL and SWRL languages could bring the representation additional work. In OWL and SWRL, expressiveness and reasoning capability are contradictory. The more a language is expressive, the less efficient and stable the reasoning is [28, 29]. To ensure the decidability of reasoning, the expressiveness of OWL and SWRL is restricted. As an example, the representation of $n$-ary relations is not directly supported in OWL and is also not available in SWRL. To use OWL/ SWRL to represent $n$-ary relations, each $n$-ary relation must be firstly transformed to some binary relations and then one can use an OWL property to represent each binary relation. Unfortunately, tolerance semantic representation sometimes requires the representation of $n$-ary relations. At these times, the transformation from $n$-ary relations to binary relations is indispensable. Such additional work is time consuming and error-prone. (2) There is a lack of a both systematic and formal methodology for constructing an OWL/SWRL ontology. Although there are various ontology construction methodologies and some of them are systematic, all of these methodologies are non-formal. This leads to a situation that different people construct different OWL/SWRL ontologies for the same tolerance specification and it is very difficult to distinguish which ontology is the best. (3) Reasoning on an OWL/ SWRL ontology is somewhat time-consuming. It has been proved that the concept satisfiability problem is NExpTimecomplete for OWL DL [50] and the problem of reasoning on OWL DL ontologies combining with DL-safe SWRL rules is ExpTime-complete [44]. For the reasoning on an ontology of the tolerance semantics of a simple component, such time complexities are acceptable. However, for the reasoning on an ontology of the tolerance semantics of a complex component or assembly, such time complexities become relatively high.

Although the DL ontology-based approach for explicitly representing tolerance semantics has some limitations, there is still a certain sense of studying the integration of this approach and commercial CAD/CAM systems from the view of academic study. Lastly, it is necessary to point out that the DL ontology based approach should not be seen as a complete replacement of the existing approaches for tolerance representation in commercial CAD/CAM systems, but more as an alternative approach to improve these approaches in some aspects.

Acknowledgments The authors would like to appreciate the insightful comments from the two anonymous reviewers for the improvement of the paper. The authors also would like to acknowledge the financial supports by the National Natural Science Foundation of China (Nos. 51475190 and 51365009), the Hubei Provincial Natural Science Foundation of China (No. 2015CFA109), the Doctoral Dissertation Innovation Foundation of Huazhong University of Science and Technology, and the National Scholarship of China Scholarship Council. 
Appendix A. DL definitions of the complex concepts in CompositePositionalTolerance

PatternOfHoles $\equiv$ RectangularPatternOfHoles

$\sqcup$ CircularPatternOfHoles

CompositeFeatureControlFrame $\equiv$

FeatureControlFrame

$\sqcap \exists$ ヨasToleranceSymbol.

PositionalToleranceSymbol

$\sqcap \exists$ hasSegment.(UpperSegment

$\sqcup$ LowerSegment)

CompositeToleranceZone $\equiv P L T Z F \sqcap F R T Z F$

UpperSegment $\equiv$ Segment

$\sqcap \exists$ GasToleranceValue.float

$\sqcap \exists$ hasTolerancePrinciple.

MaximumMaterialCondition

$\sqcap \exists$ GasPrimaryDatum.PrimaryDatum

$\sqcap \exists$ hasSecondaryDatum.SecondaryDatum

$\sqcap \exists$ hasSecondaryDatumPrinciple.

MaximumMaterialCondition

$\sqcap \exists$ hasTertiaryDatum.TertiaryDatum

$\sqcap \exists$ GasTertiaryDatumPrinciple.

MaximumMaterialCondition

LowerSegment $\equiv$ Segment

$\sqcap \exists$ hasToleranceValue.float

$\sqcap \exists$ hasTolerancePrinciple.

MaximumMaterialCondition

$\sqcap$ ( $\exists$ hasPrimaryDatum.PrimaryDatum $\sqcup$

( $\exists$ hasPrimaryDatum.PrimaryDatum

$\sqcap \exists$ hasSecondaryDatum.SecondaryDatum

$\sqcap \exists$ hasSecondaryDatumPrinciple.

MaximumMaterialCondition)

$\sqcup$ ( $\exists$ hasPrimaryDatum.PrimaryDatum

$\sqcap \exists$ hasSecondaryDatum.SecondaryDatum

$\sqcap \exists$ hasSecondaryDatumPrinciple.

MaximumMaterialCondition

$\sqcap \exists$ GasTertiaryDatum.TertiaryDatum

$\sqcap \exists$ hasTertiaryDatumPrinciple.

MaximumMaterialCondition))
Appendix B. Horn rules defining the constraint relationships in the $\mathrm{CPT}$ ontology

hasMeetMaximumMaterialCondition $\left(t_{1}, m m c\right)$

$\leftarrow$ PositionalTolerance $\left(t_{1}\right)$,

MaximumMaterialCondition $(\mathrm{mmc})$,

$\operatorname{Hole}\left(C_{1,1}\right)$, Size (efs),

isExternalFunctionSizeOf(efs, $\left.C_{1,1}\right)$,

isGreaterThanOrEqualTo (efs, $\left.\phi\left(t-l-t_{1}\right)\right)$,

Size(las), isLocalActualSizeOf(las, $\left.C_{1,1}\right)$,

isGreaterThanOrEqualTo(las, $\phi(t-l))$,

isSmallerThanOrEqualTo (las, $\phi(t+u))$

isPLTZFOf $\left(x_{4}, x_{1}\right)$

$\leftarrow$ CompositePositionalTolerance $\left(x_{1}\right)$,

PatternOfHoles $(H)$,

hasTolerancedFeature $\left(x_{1}, H\right)$,

CompositeFeatureControlFrame $\left(x_{2}\right)$,

hasFeature ControlFrame $\left(x_{1}, x_{2}\right)$,

UpperSegment $\left(x_{3}\right)$, hasSegment $\left(x_{2}, x_{3}\right)$,

$\operatorname{PLTZF}\left(x_{4}\right)$, hasEstablish $\left(x_{3}, x_{4}\right)$,

CylindricalToleranceZone $\left(x_{5}\right)$,

hasContain $\left(x_{4}, x_{5}\right)$, hasDiameter $\left(x_{5}, t_{1}\right)$,

CylindricalToleranceZone $\left(x_{6}\right)$,

hasContain $\left(x_{4}, x_{6}\right)$, hasDiameter $\left(x_{6}, t_{1}\right)$,

CylindricalToleranceZone $\left(x_{7}\right)$,

hasContain $\left(x_{4}, x_{7}\right)$, hasDiameter $\left(x_{7}, t_{1}\right)$,

CylindricalToleranceZone $\left(x_{8}\right)$,

hasContain $\left(x_{4}, x_{8}\right)$, hasDiameter $\left(x_{8}, t_{1}\right)$,

CylindricalToleranceZone $\left(x_{9}\right)$,

hasContain $\left(x_{4}, x_{9}\right)$, hasDiameter $\left(x_{9}, t_{1}\right)$,

CylindricalToleranceZone $\left(x_{10}\right)$,

hasContain $\left(x_{4}, x_{10}\right)$, hasDiameter $\left(x_{10}, t_{1}\right)$,

MaximumMaterialCondition $\left(x_{11}\right)$,

hasMeetMaximumMaterialCondition $\left(x_{3}, x_{11}\right)$,

PrimaryDatum(A),hasPrimaryDatum $\left(x_{3}, \mathrm{~A}\right)$,

SecondaryDatum(B), hasSecondaryDatum $\left(x_{3}, \mathrm{~B}\right)$,

TertiaryDatum $(\mathrm{C})$, hasTertiaryDatum $\left(x_{3}, \mathrm{C}\right)$,

hasPerpendicular $\left(x_{5}, \mathrm{~A}\right)$, hasPerpendicular $\left(x_{6}, \mathrm{~A}\right)$, hasPerpendicular $\left(x_{7}, \mathrm{~A}\right)$, hasPerpendicular $\left(x_{8}, \mathrm{~A}\right)$, hasPerpendicular $\left(x_{9}, \mathrm{~A}\right)$, hasPerpendicular $\left(x_{10}, \mathrm{~A}\right)$, Distance $\left(x_{12}\right)$, hasPrimaryObject $\left(x_{12}, x_{8}\right)$,

hasSecondaryObject $\left(x_{12}, \mathrm{~B}\right)$,

has Value $\left(x_{12}, d_{2}\right)$, Distance $\left(x_{13}\right)$,

hasPrimaryObject $\left(x_{13}, x_{8}\right)$,

hasSecondaryObject $\left(x_{13}, \mathrm{~B}\right)$, hasValue $\left(x_{13}, d_{3}\right)$

MeetRequirementOfCPT $\left(x_{2}, x_{1}\right)$

$\leftarrow$ CompositePositionalTolerance $\left(x_{1}\right)$, 
CompositeVariationalGeometry $\left(x_{2}\right)$, has VariationalGeometry $\left(x_{1}, x_{2}\right), \operatorname{PLTZF}\left(x_{3}\right)$, isPLTZFOf $\left(x_{3}, x_{1}\right), F R T Z F\left(x_{4}\right)$, isFRTZFOf $\left(x_{4}, x_{1}\right)$, Intersection $\left(x_{5}\right)$, hasPrimaryObject $\left(x_{5}, x_{3}\right)$, hasSecondaryObject $\left(x_{5}, x_{4}\right)$, hasResultantToleranceZone $\left(x_{1}, x_{5}\right)$, hasFallinside $\left(x_{2}, x_{5}\right)$

\section{Appendix C. DL assertional axioms of the facts depicts in Fig. 2}

CompositePositionalTolerance(tol), RectangularPatternOfHoles $\left(\mathrm{H}_{1}-\mathrm{H}_{2}-\mathrm{H}_{3}-\mathrm{H}_{4}-\mathrm{H}_{5}-\mathrm{H}_{6}\right)$, hasTolerancedFeature(tol, $\mathrm{H}_{1}-\mathrm{H}_{2}-\mathrm{H}_{3}-\mathrm{H}_{4}-\mathrm{H}_{5}-\mathrm{H}_{6}$ ), CompositeFeatureControlFrame (cfcf), hasFeatureControlFrame(tol, cfcf), PositionalToleranceSymbol(pts), hasToleranceSymbol(cfcf, pts), UpperSegment(upseg), hasSegment(cfff, upseg), LowerSegment(lowseg), hasSegment(cfcf, lowseg), hasToleranceValue(upseg, $t_{1}$ ), MaximumMaterialCondition $(m m c)$, hasTolerancePrinciple(upseg, mmc), PrimaryDatum(A),hasPrimaryDatum(upseg, A), SecondaryDatum(B), hasSecondaryDatum (upseg, B), TertiaryDatum(C), hasTertiaryDatum(upseg, C), hasToleranceValue(lowseg, $t_{2}$ ), hasTolerancePrinciple(lowseg, mmc), hasPrimaryDatum(lowseg, A)

\section{Appendix D. DL assertional axioms of the resultant tolerance zone in Fig. 4}

\section{$\operatorname{PLTZF}\left(C_{1, j}\right), \operatorname{FRTZF}\left(C_{2, j}\right)$,}

CompositeToleranceZone $\left(Z_{j}\right)$,

hasResultantToleranceZone (tol, $\left.Z_{j}\right)$,

CompositeVariationalGeometry $(V)$,

has VariationalGeometry (tol, V), hasDiameter $\left(C_{i, j}, t_{i}\right)$, hasPerpendicular $\left(C_{i, j}, \mathrm{~A}\right)$, hasFallInside $\left(V, Z_{j}\right)$,

Distance $\left(D_{1}\right)$, hasPrimaryObject $\left(D_{1}, A_{1,1}\right)$, hasSecondaryObject $\left(D_{1}, A_{1,2}\right)$, hasValue $\left(D_{1}, d_{4}\right)$, $\operatorname{Distance}\left(D_{2}\right)$, hasPrimaryObject $\left(D_{2}, A_{1,1}\right)$, hasSecondaryObject $\left(D_{2}, A_{1,4}\right)$, hasValue $\left(D_{2}, d_{1}\right)$, Distance $\left(D_{3}\right)$, hasPrimaryObject $\left(D_{3}, A_{1,1}\right)$, hasSecondaryObject $\left(D_{3}, \mathrm{C}\right)$, hasValue $\left(D_{3}, d_{3}\right)$, Distance $\left(D_{4}\right)$, hasPrimaryObject $\left(D_{4}, A_{1,2}\right)$, hasSecondaryObject $\left(D_{4}, A_{1,3}\right)$, hasValue $\left(D_{4}, d_{5}\right)$, $\operatorname{Distance}\left(D_{5}\right)$, hasPrimaryObject $\left(D_{5}, A_{1,2}\right)$, hasSecondaryObject $\left(D_{5}, A_{1,5}\right)$, hasValue $\left(D_{5}, d_{1}\right)$, Distance $\left(D_{6}\right)$, hasPrimaryObject $\left(D_{6}, A_{1,3}\right)$, hasSecondaryObject $\left(D_{6}, A_{1,6}\right)$, has Value $\left(D_{6}, d_{1}\right)$, Distance $\left(D_{7}\right)$, hasPrimaryObject $\left(D_{7}, A_{1,4}\right)$, hasSecondaryObject $\left(D_{7}, A_{1,5}\right)$, has Value $\left(D_{7}, d_{4}\right)$,
$\operatorname{Distance}\left(D_{8}\right)$, hasPrimaryObject $\left(D_{8}, A_{1,4}\right)$, hasSecondaryObject $\left(D_{8}, \mathrm{~B}\right)$, hasValue $\left(D_{8}, d_{2}\right)$, Distance $\left(D_{9}\right)$, hasPrimaryObject $\left(D_{9}, A_{1,4}\right)$, hasSecondaryObject $\left(D_{9}, \mathrm{C}\right)$, hasValue $\left(D_{9}, d_{3}\right)$, Distance $\left(D_{10}\right)$, hasPrimaryObject $\left(D_{10}, A_{1,5}\right)$, hasSecondaryObject $\left(D_{10}, A_{1,6}\right)$, hasValue $\left(D_{10}, d_{5}\right)$, Distance $\left(D_{11}\right)$, hasPrimaryObject $\left(D_{11}, A_{1,5}\right)$, hasSecondaryObject $\left(D_{11}, \mathrm{~B}\right)$, has Value $\left(D_{11}, d_{2}\right)$, Distance $\left(D_{12}\right)$, hasPrimaryObject $\left(D_{12}, A_{1,6}\right)$, hasSecondaryObject $\left(D_{12}, \mathrm{~B}\right)$, hasValue $\left(D_{12}, d_{2}\right)$, Distance $\left(D_{13}\right)$, hasPrimaryObject $\left(D_{13}, A_{2,1}\right)$, hasSecondaryObject $\left(D_{13}, A_{2,2}\right)$, hasValue $\left(D_{13}, d_{4}\right)$, Distance $\left(D_{14}\right)$, hasPrimaryObject $\left(D_{14}, A_{2,1}\right)$, hasSecondaryObject $\left(D_{14}, A_{2,4}\right)$, hasValue $\left(D_{14}, d_{1}\right)$, Distance $\left(D_{15}\right)$, hasPrimaryObject $\left(D_{15}, A_{2,2}\right)$, hasSecondaryObject $\left(D_{15}, A_{2,3}\right)$, hasValue $\left(D_{15}, d_{5}\right)$, Distance $\left(D_{16}\right)$, hasPrimaryObject $\left(D_{16}, A_{2,2}\right)$, hasSecondaryObject $\left(D_{16}, A_{2,5}\right)$, hasValue $\left(D_{16}, d_{1}\right)$, Distance $\left(D_{17}\right)$, hasPrimaryObject $\left(D_{17}, A_{2,3}\right)$, hasSecondaryObject $\left(D_{17}, A_{2,6}\right)$, hasValue $\left(D_{17}, d_{1}\right)$, Distance $\left(D_{18}\right)$, hasPrimaryObject $\left(D_{18}, A_{2,4}\right)$, hasSecondaryObject $\left(D_{18}, A_{2,5}\right)$, hasValue $\left(D_{18}, d_{4}\right)$, Distance $\left(D_{19}\right)$, hasPrimaryObject $\left(D_{19}, A_{2,5}\right)$, hasSecondaryObject $\left(D_{19}, A_{2,6}\right)$, hasValue $\left(D_{19}, d_{5}\right)$, where $i=1,2, j=1,2, \ldots, 6, C_{i, j}=\left\{(x, y) \mid x^{2}+y^{2} \leq t_{i}^{2}\right\}$, and $A_{i, j}$ is the axis of $C_{i, j}$.

Open Access This article is distributed under the terms of the Creative Commons Attribution 4.0 International License (http:// creativecommons.org/licenses/by/4.0/), which permits unrestricted use, distribution, and reproduction in any medium, provided you give appropriate credit to the original author(s) and the source, provide a link to the Creative Commons license, and indicate if changes were made.

\section{References}

1. Armillotta A (2013) A method for computer-aided specification of geometric tolerances. Comput Aided Des 45(12):1604-1616

2. ISO 1101 (2012) Geometrical product specifications (GPS) - geometrical tolerancing - tolerances of form, orientation, location and run-out. International Organization for Standardization, Geneva

3. ASME Y14.5 (2009) Dimensioning and tolerancing. American Society of Mechanical Engineers, New York

4. Turner JU, Wozny MJ (1987) Tolerances in computer-aided geometric design. The Visual Comput 3(4):214-226

5. Gossard DC, Zuffante RP, Sakurai H (1988) Representing dimensions, tolerances, and features in MCAE systems. IEEE Comput Graph Appl 8(2):51-59

6. Requicha AAG, Chan SC (1986) Representation of geometric features, tolerances, and attributes in solid modelers based on constructive geometry. IEEE J Robot Autom 2(3):156-166

7. Roy U, Liu CR (1988) Feature-based representational scheme of a solid modeler for providing dimensioning and tolerancing information. Robot Comput-Integr Manuf 4(3):335-345 
8. Martinsen K (1993) Vectorial tolerancing for all types of surfaces. Proc. 19th ASME Des. Autom. Conf., p 187-198

9. Rivest L, Fortin C, Morel C (1994) Tolerancing a solid model with a kinematic formulation. Comput Aided Des 26(6):465-476

10. Chase KW, Gao J, Magleby SP, Sorenson CD (1996) Including geometric feature variations in tolerance analysis of mechanical assemblies. IIE Trans 28(10):795-808

11. Desrochers A, Clemént A (1994) A dimensioning and tolerancing assistance model for CAD/CAM systems. Int J Adv Manuf Technol 9(6):352-361

12. Desrochers A (1999) Modeling three dimensional tolerance zones using screw parameters. Proc. 25th ASME Des. Autom. Conf., p 895-904

13. Kandikjan T, Shah JJ, Davidson JK (2001) A mechanism for validating dimensioning and tolerancing schemes in CAD systems. Comput Aided Des 33(10):721-737

14. Davidson JK, Mujezinovic A, Shah JJ (2002) A new mathematical model for geometric tolerances as applied to round faces. ASME Trans J Mech Des 124(4):609-622

15. Mujezinovic A, Davidson JK, Shah JJ (2004) A new mathematical model for geometric tolerances as applied to polygonal faces. ASME Trans J Mech Des 126(3):504-518

16. Ameta G, Davidson JK, Shah JJ (2007) Tolerance-maps applied to a point-line cluster of features. ASME Trans J Mech Des 129(8): $782-792$

17. ISO 10303-203 (2011) Industrial automation systems and integration - product data representation and exchange - part 203: application protocol: configuration controlled 3D design of mechanical parts and assemblies. International Organization for Standardization, Geneva

18. ISO 10303-214 (2010) Industrial automation systems and integration - product data representation and exchange - part 214: application protocol: core data for automotive mechanical design processes. International Organization for Standardization, Geneva

19. ISO 10303-242 (2014) Industrial automation systems and integration - product data representation and exchange - part 242: application protocol: managed model-based 3D engineering. International Organization for Standardization, Geneva

20. Sarigecili MI, Roy U, Rachuri S (2014) Interpreting the semantics of GD\&T specifications of a product for tolerance analysis. Comput Aided Des 47(2):72-84

21. Feeney AB, Frechette SP, Srinivasan V (2015) A portrait of an ISO STEP tolerancing standard as an enabler of smart manufacturing systems. ASME Trans J Comput Inf Sci Eng 15(2):021001

22. Zhong Y, Qin Y, Huang M, Lu W, Chang L (2014) Constructing a meta-model for assembly tolerance types with a description logic based approach. Comput Aided Des 48(3):1-16

23. Qin Y, Lu W, Liu X, Huang M, Zhou L, Jiang X (2015) Description logic-based automatic generation of geometric tolerance zones. Int $\mathrm{J}$ Adv Manuf Tech 79(5):1221-1237

24. Lu W, Qin Y, Liu X, Huang M, Zhou L, Jiang X (2015) Enriching the semantics of variational geometric constraint data with ontology. Comput Aided Des 63(6):72-85

25. Liu Y, Gao S, Cao Y (2009) An efficient approach to interpreting rigorous tolerance semantics for complicated tolerance specification. IEEE Trans Autom Sci Eng 6(4):670-684

26. Baader F, Calvanese D, McGuinness DL, Nardi D, Patel-Schneider PF (2010) The description logic handbook: theory, implementation and applications, 2nd edn. Cambridge University Press, Cambridge

27. Horn A (1951) On sentences which are true of direct unions of algebras. J Symbolic Logic 16(1):14-21

28. McGuinness DL, van Harmelen F (2004) OWL Web Ontology Language Overview W3C Recommendation. http://www.w3. org/TR/owl-features/
29. Horrocks I, Patel-Schneider PF, Boley H, Tabet S, Grosof B, Dean M (2004) SWRL: a semantic web rule language combining OWL and RuleML. http://www.w3.org/Submission/SWRL/

30. Srinivasan V (2008) Standardizing the specification, verification, and exchange of product geometry: research, status and trends. Comput Aided Des 40(7):738-749

31. ISO 10303-1 (1994) Industrial automation systems and integration - product data representation and exchange - part 1: overview and fundamental principles. International Organization for Standardization, Geneva

32. ISO 10303-11 (2004) Industrial automation systems and integration - product data representation and exchange - part 11: description methods: the EXPRESS language reference manual. International Organization for Standardization, Geneva

33. Rachuri S, Han YH, Feng SC, Roy U, Wang F, Sriram RD, Lyons KW (2004) Object-oriented representation of electro-mechanical assemblies using UML, NISTIR 7057. National Institute of Standards and Technology, Gaithersburg

34. Zhao X, Pasupathy TK, Wilhelm RG (2006) Modeling and representation of geometric tolerances information in integrated measurement processes. Comput Ind 57(4):319-330

35. Dantan JY, Ballu A, Mathieu L (2008) Geometrical product specifications - model for product life cycle. Comput Aided Des 40(4): 493-501

36. Ballu A, Mathieu L, Dantan JY (2015) Formal language for GeoSpelling. ASME Trans J Comput Inf Sci Eng 15(2): 021002

37. Lu W, Jiang X, Liu X, Qi Q, Scott PJ (2010) Modeling the integration between specifications and verification for cylindricity based on category theory. Meas Sci Tech 21(11):115107

38. Xu Y, Xu Z, Jiang X, Scott PJ (2011) Developing a knowledgebased system for complex geometrical product specification (GPS) data manipulation. Knowl-Based Syst 24(1):10-22

39. Qi Q, Scott PJ, Jiang X, Lu W (2014) Design and implementation of an integrated surface texture information system for design, manufacture and measurement. Comput Aided Des 57(12):41-53

40. Fiorentini X, Gambino I, Liang VC, Foufou S, Rachuri R, Mani M, Bock C (2007) An ontology for assembly representation, NISTIR 7436. National Institute of Standards and Technology, Gaithersburg

41. Ahmed F, Han S (2015) Interoperability of product and manufacturing information using ontology. Concurrent Eng Res Appl 23(3): 265-278

42. Liu Y, Gao S (2004) Variational geometry based pre-inspection of a pattern of holes. Int J Prod Res 42(8):1659-1675

43. Liu Y, Gao S (2006) Generating variational geometry of a hole with composite tolerances. IEEE Trans Autom Sci Eng 3(1):92-107

44. Ortiz M, Rudolph S, Simkus M (2010) Worst-case optimal reasoning for the Horn-DL FRAgments of OWL 1 and 2. Proc. 12th Int. Conf. Prin. Knowl. Representation Reasoning, p 269-279

45. Abdul-Ghafour S, Ghodous P, Shariat B, Perna E, Khosrowshahi F (2014) Semantic interoperability of knowledge in feature-based CAD models. Comput Aided Des 56(11):45-57

46. Stanford Center for Biomedical Informatics Research (2012) Protégé:3.5 http://protege.stanford.edu/

47. Friedman-Hill E (2003) Jess in action: rule-based Systems in Java. Manning Publications, Greenwich

48. Ciocoiu M, Nau DS, Gruninger M (2001) Ontologies for integrating engineering applications. ASME Trans J Comput Inf Sci Eng 1(1): 12-22

49. Qin Y, Lu W, Qi Q, Liu X, Zhong Y, Scott PJ, Jiang X (2016) Status, comparison, and issues of CAD model data exchange methods based on standardized neutral files and OWL file. ASME Trans J Comput Inf Sci Eng. doi:10.1115/1.4034325

50. Zolin E (2013) Complexity of reasoning in description logics. http://www.cs.man.ac.uk/ ezolin/dl/ 